Article

\title{
On the Design of an Integrated System for Wave Energy Conversion Purpose with the Reaction Mass on Board
}

\author{
Jinming $\mathrm{Wu}^{*}$ and Zhonghua $\mathrm{Ni}$ \\ School of Mechanical Engineering, Southeast University, Nanjing 211189, China; nzh2003@seu.edu.cn \\ * Correspondence: jinmingwu@seu.edu.cn;
}

Received: 12 March 2020; Accepted: 2 April 2020; Published: 3 April 2020

\begin{abstract}
In this paper, we investigate the design of an integrated system consisting of two non-rigidly connected bodies: A floating buoy and an emerged offshore structure. When waves excite the buoy to oscillate, the relative motion between the two bodies are converted to useful energy through a spring damper system, resulting in wave energy being absorbed. The parameter to design includes the mass and underwater shape of the buoy. The spring stiffness of the power take-off (PTO) system is constrained to be non-negative with the concerns of complexity in implementation and system stability. Results suggest that a larger mass of the buoy is advantageous due to smaller optimal spring stiffness and damping coefficient of the PTO system, more absorbed wave power, and less motion amplitude of the two bodies. A favorable underwater shape of the buoy is characterized by large diameter to draft ratio, with the section profile preferring a circle or square rather than an equilateral triangle. Investigations on the designed buoy in irregular waves show that the integrated system presents its peak power absorption within the common range of energy period, and the motion amplitude of the offshore structure is larger than the wave amplitude in a certain range of sea states.
\end{abstract}

Keywords: integrated system; floating buoy; offshore structure; wave energy converter; non-negative spring stiffness

\section{Introduction}

Due to high energy density and excellent predictability, ocean wave energy stands up as a promise substitute of fossil energies, which give rise to environmental issues. Although the first wave energy converter (WEC) had been patented over two centuries ago [1], wave energy conversion technology is still immature and at a pre-commercialized stage. The levelized cost of wave energy is estimated to be $\$ 1.0 \sim 1.5 / \mathrm{kWh}$, and not ready for the present power economic request when compared to $\$ 0.07 / \mathrm{kWh}$ of onshore wind energy [2]. A possible solution to minimize this discrepancy is to combine WECs with offshore structures, such as navigation aids [3,4], offshore solar [5] and wind [6] electricity generation systems, and even offshore aquaculture devices [2], so that the substructure, mooring system, and electrical grid connections, which account for respectively $20 \%, 10 \%$, and $6 \%$ of the construction cost of an individual WEC [7], can be shared.

In view of wave energy absorption, the aforementioned integrated systems belong to the two-body type, which uses the relative motion between two bodies to harvest energy. A two-body system usually consists of two parts: A floating buoy that moves along with the incoming wave and a reaction mass, which can be submerged in deep water [8,9] or on board [10]. A two-body system with the reaction mass on board has the advantage of lacking submerged moving bodies and actuators, and may be easier for the installation and operation process [10]. Studies on the two-body system with reaction mass on board can be traced back to Parks [11]. French et al. [12] applied phase control to a 
heaving buoy reacting against an internal mass. Korde [10] revealed that the on board system shows a power capture width ratio comparable with that of the submerged system over a wide range of wave frequency, demonstrating its potential in wave power absorption. Additionally, Korde [13] proposed a motion compensation system to achieve stationarity of the reaction mass to further increase the absorbed power for the on board system.

As concerns how to integrate WECs with offshore structures to form a two-body system, one option is that the offshore structure be on board a floating buoy, which is demonstrated in [14] that a multi-purpose platform is on board floating buoyancy columns with actuators connecting them to absorb wave energy. In order to achieve the best wave power absorption performance, previous studies on two-body systems with reaction mass on board tried to optimize the parameter of the reaction mass $[10-13,15]$. However, in the integrated system of the offshore structure and the floating buoy, the reaction mass (the offshore structure) is normally provided as fixed parameters ahead of time, hence only the parameter of the floating buoy can be optimized.

The objective of this paper is to design the parameter of the floating buoy with an offshore structure on board to achieve satisfied performance of the integrated system. Here, the parameter of the floating buoy consists of its mass and underwater shape, while the performance of the integrated system includes absorbed wave power and motion amplitude of the two bodies. A $5 \mathrm{MW}$ offshore wind turbine is chosen as the offshore structure for a case study. When WECs are resonant with waves, the absorbed wave power reaches the maximum. However, in order to achieve resonance, the optimal spring stiffness of the power take-off (PTO) system may sometimes be negative, which not only is difficult to implement in reality, but may also shift WECs to unsteady states. Therefore, the spring stiffness of the PTO system is constrained to be non-negative in this paper. The designing process is performed in regular waves, and the designed integrated system is then investigated in irregular waves.

\section{Methods}

Figure 1 shows the integrated two-body system consisting of the offshore structure and the floating buoy, and with the offshore structure on board the floating buoy. The PTO system makes use of the relative motion between the two bodies to produce useful power, and is simplified as a pair of spring and damper connected in parallel. The stiffness of the spring and the damping coefficient of the damper are denoted as $k_{p}$ and $c_{p}$, respectively. The mass and displacement of the offshore structure are respectively denoted as $m_{s}$ and $z_{s}$, and that of the buoy as $m_{b}$ and $z_{b}$. For simplicity, the offshore structure and the buoy are subjected to heave motion only.

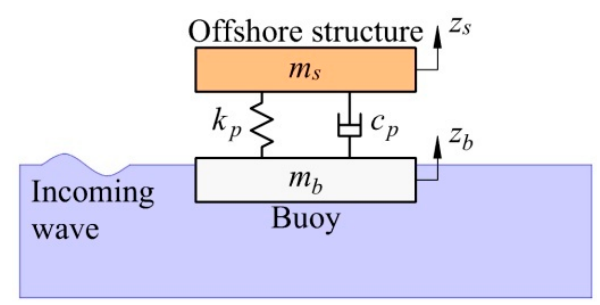

Figure 1. Schematic diagram of the integrated system.

In regular waves, according to the fundamental investigation on two-body systems by Falnes [16], the equation of motion of the offshore structure and the buoy can be written as

$$
\begin{gathered}
m_{s} \ddot{z}_{s}+c_{p}\left(\dot{z}_{s}-\dot{z}_{b}\right)+k_{p}\left(z_{s}-z_{b}\right)=0 ; \\
{\left[m_{b}+m_{b h}(\omega)\right] \ddot{z}_{b}+c_{b h}(\omega) \dot{z}_{b}+k_{b h} z_{b}-c_{p}\left(\dot{z}_{s}-\dot{z}_{b}\right)-k_{p}\left(z_{s}-z_{b}\right)=f_{d}}
\end{gathered}
$$

where $m_{b h}, c_{b h}$, and $k_{b h}$ denote the added mass, radiation damping coefficient, and hydrostatic stiffness of the buoy, $\omega$ denotes the wave frequency, and $f_{d}$ denotes the excitation force the incoming wave acts 
on the buoy. The hydrodynamic coefficients and excitation force are calculated numerically by the ANSYS AQWA software [17]. The hydrostatic stiffness of the buoy in heave is calculated as

$$
k_{b h}=\rho g S,
$$

where $\rho$ is the water density, $g$ is the gravity acceleration, and $S$ is the area of the waterplane. By applying the form of complex amplitude [18,19], which is denoted by a symbol "^ on top of the variable, Equation (1) can be written as

$$
\begin{gathered}
-\omega^{2} m_{s} \hat{z}_{s}+\left(k_{p}+\mathrm{i} \omega c_{p}\right)\left(\hat{z}_{s}-\hat{z}_{b}\right)=0 \\
-\omega^{2}\left[m_{b}+m_{b h}(\omega)\right] \hat{z}_{b}+\left[k_{b h}+\mathrm{i} \omega c_{b h}(\omega)\right] \hat{z}_{b}-\left(k_{p}+\mathrm{i} \omega c_{p}\right)\left(\hat{z}_{s}-\hat{z}_{b}\right)=\hat{f}_{d}
\end{gathered}
$$

Equation (3) can be transferred to the matrix form as

$$
\left[\begin{array}{ll}
-\omega^{2} m_{s}+k_{p}+\mathrm{i} \omega c_{p} & -\left(k_{p}+\mathrm{i} \omega c_{p}\right) \\
-\left(k_{p}+\mathrm{i} \omega c_{p}\right) & -\omega^{2}\left[m_{b}+m_{b h}(\omega)\right]+k_{b h}+\mathrm{i} \omega c_{b h}(\omega)+k_{p}+\mathrm{i} \omega c_{p}
\end{array}\right]\left[\begin{array}{c}
\hat{z}_{s} \\
\hat{z}_{b}
\end{array}\right]=\left[\begin{array}{c}
0 \\
\hat{f}_{d}
\end{array}\right] .
$$

Here, we denote the impedance of the offshore structure, the buoy, and the PTO system as $Z_{s}, Z_{b}$, and $Z_{p}$, and their real and imaginary parts are denoted by $Z_{s r}, \mathrm{i} Z_{s i}, Z_{b r}, \mathrm{i} Z_{b i}, Z_{p r}$, and i $Z_{p i}$, respectively. The impedance is defined as

$$
\begin{gathered}
Z_{s r}=-\omega^{2} m_{s} \\
Z_{b r}=-\omega^{2}\left[m_{b}+m_{b h}(\omega)\right]+k_{b h} ; \\
Z_{b i}=\omega c_{b h}(\omega) \\
Z_{p r}=k_{p} \\
Z_{p i}=\omega c_{p} .
\end{gathered}
$$

Since the offshore structure is out of water, $Z_{s i}=0$. Then, Equation (4) can be written as

$$
\left[\begin{array}{ll}
Z_{s}+Z_{p} & -Z_{p} \\
-Z_{p} & Z_{b}+Z_{p}
\end{array}\right]\left[\begin{array}{l}
\hat{z}_{s} \\
\hat{z}_{b}
\end{array}\right]=\left[\begin{array}{l}
0 \\
\hat{f}_{d}
\end{array}\right]
$$

By finding the inverse of the matrix in the left term of Equation (6) and matrix multiplication, it gives

$$
\left[\begin{array}{l}
\hat{z}_{s} \\
\hat{z}_{b}
\end{array}\right]=\frac{1}{Z_{s} Z_{b}+\left(Z_{s}+Z_{b}\right) Z_{p}}\left[\begin{array}{c}
Z_{p} \hat{f}_{d} \\
\left(Z_{s}+Z_{p}\right) \hat{f}_{d}
\end{array}\right]
$$

For a two-body system, the time-averaged absorbed power can be calculated by [20]

$$
P=\frac{\omega^{2} c_{p}}{2}\left|\hat{z}_{s}-\hat{z}_{b}\right|^{2}
$$

It can be obtained from Equation (7) that

$$
\hat{z}_{s}-\hat{z}_{b}=\frac{-\hat{f}_{d}}{Z_{b}+\left(1+\frac{Z_{b}}{Z_{s}}\right) Z_{p}} .
$$

By denoting

$$
G=1+\frac{Z_{b}}{Z_{s}}
$$

Equation (9) can be written as

$$
\hat{z}_{s}-\hat{z}_{b}=\frac{-\hat{f}_{d}}{Z_{b}+G Z_{p}} .
$$


Here, we denote the real and imaginary part of $G$ as $G_{r}$ and $i G_{i}$, respectively. Then, we have

$$
\begin{gathered}
G_{r}=1+\frac{Z_{b r}}{Z_{s r}} \\
G_{i}=\frac{Z_{b i}}{Z_{s r}}
\end{gathered}
$$

Inserting Equation (11) to Equation (8) gives

$$
P=\frac{\omega^{2} c_{p}}{2} \frac{\hat{f}_{d} \hat{f}_{d}^{*}}{\left(Z_{b}+G Z_{p}\right)\left(Z_{b}^{*}+G^{*} Z_{p}^{*}\right)} .
$$

Decomposition of the complex parameters to real and imaginary parts makes Equation (13) be written as

$$
P=\frac{\omega}{2} \frac{\left|\hat{f_{d}}\right|^{2}}{|G|^{2} Z_{p i}+2\left(G_{r} Z_{b i}-G_{i} Z_{b r}\right)+\frac{|G|^{2} Z_{p r}^{2}+2\left(G_{r} Z_{b r}+G_{i} Z_{b i}\right) Z_{p r}+\left|Z_{b}\right|^{2}}{Z_{p i}}}
$$

When there is no constraint on the parameters of the PTO system, it can be seen that when

$$
Z_{p r}=Z_{p r o}=-\frac{G_{r} Z_{b r}+G_{i} Z_{b i}}{|G|^{2}}
$$

And

$$
Z_{p i}=Z_{p i o}=\frac{G_{r} Z_{b i}-G_{i} Z_{b r}}{|G|^{2}}
$$

the absorbed power reaches the maximum

$$
P_{\max }=\frac{\omega}{8} \frac{\left|\hat{f_{d}}\right|^{2}}{G_{r} Z_{b i}-G_{i} Z_{b r}} .
$$

Equation (5) and Equation (12) gives

$$
G_{r} Z_{b i}-G_{i} Z_{b r}=\left(1+\frac{Z_{b r}}{Z_{s r}}\right) Z_{b i}-\frac{Z_{b i}}{Z_{s r}} Z_{b r}=Z_{b i}
$$

From Equation (15) to Equation (18) and the relation between $Z_{p r}, Z_{p i}$, and $k_{p}, c_{p}$ as shown in Equation (5), we can obtain the optimal values of $k_{p}, c_{p}$, and maximum absorbed power as

$$
\begin{gathered}
k_{p o}=-\frac{G_{r} Z_{b r}+G_{i} Z_{b i}}{|G|^{2}} \\
c_{p o}=\frac{c_{b h}}{|G|^{2}} ; \\
P_{\max }=\frac{\left|\hat{f}_{d}\right|^{2}}{8 c_{b h}} .
\end{gathered}
$$

It agrees with the finding of Korde [10] that the maximum absorbed power only depends on the excitation force and radiation damping coefficient of the buoy for an on board system. It is interesting to find that although the integrated system oscillates in two degrees of freedom, it behaves as a single-degree-of-freedom system in terms of power absorption.

However, in some cases, Equation (19) gives the optimal spring stiffness of the PTO system a negative value, which is not only difficult to implement in reality, but also may offset the system to unsteadiness. In this paper, we constrain the spring stiffness of the PTO system to be non-negative. 
Taking into consideration this constraint, we update the optimal spring stiffness and damping coefficient of the PTO system and the maximum absorbed power of the integrated system as

$$
\begin{gathered}
k_{p o}=\max \left\{-\frac{G_{r} Z_{b r}+G_{i} Z_{b i}}{|G|^{2}}, 0\right\}, \\
c_{p o}=\left\{\begin{array}{c}
\frac{c_{b h}}{|G|^{2}}, \text { if } k_{p o}>0 \\
\left|\frac{Z_{b}}{\omega G}\right|, \text { if } k_{p o}=0
\end{array}\right.
\end{gathered}
$$

And

$$
P_{\max }= \begin{cases}\frac{\left|\hat{f}_{d}\right|^{2}}{8 c_{b h}}, & \text { if } k_{p o}>0 \\ \frac{\omega}{4} \frac{\left|\hat{f}_{d}\right|^{2}}{G|| Z_{b} \mid+Z_{b i}}, & \text { if } k_{p o}=0\end{cases}
$$

In this paper, a $5 \mathrm{MW}$ offshore wind turbine, which is used in the WindFloat concept [21] proposed by the Principle Power Inc. for offshore wind farm application, is chosen as the offshore structure for a case study. The offshore structure is restricted to oscillate in heave mode. The total mass of the offshore structure is found to be $854 \mathrm{mt}$ [21]. The water depth is considered in offshore scenario to be $h=100 \mathrm{~m}$, and the wave amplitude, which is denoted by $A$, is fixed at $1 \mathrm{~m}$ for regular waves.

\section{Effect of the Constraint on The Spring Stiffness of the PTO System}

Since the spring stiffness of the PTO system is constrained to be non-negative, which is not considered in previous literature for the on board two-body system, we investigate the effect of the constraint first. From Equation (19), we find that it is the numerator of the right term that causes the unconstrained optimal spring stiffness of the PTO system to be negative since the denominator is absolutely non-negative. Here we denote

$$
H=-\left(G_{r} Z_{b r}+G_{i} Z_{b i}\right)
$$

Inserting Equation (12) to Equation (25) gives

$$
H=-\frac{1}{Z_{s r}}\left(Z_{b r}^{2}+Z_{s r} Z_{b r}+Z_{b i}^{2}\right)
$$

According to the characteristic of parabolic functions, we find that $H$ will be negative only when

$$
Z_{s r}^{2}-4 Z_{b i}^{2} \geq 0
$$

i.e.,

$$
\frac{Z_{b i}}{\left|Z_{s r}\right|} \leq \frac{1}{2}
$$

and

$$
\frac{Z_{b r}}{\left|Z_{s r}\right|} \rightarrow \frac{1}{2}, \frac{Z_{b r}}{\left|Z_{s r}\right|} \in\left[\frac{1}{2}-\sqrt{\frac{1}{4}-\left(\frac{Z_{b i}}{\left|Z_{s r}\right|}\right)^{2}}, \frac{1}{2}+\sqrt{\frac{1}{4}-\left(\frac{Z_{b i}}{\mid Z_{s r}}\right)^{2}}\right]
$$

Here, $Z_{b r}|| Z_{s r} \mid$ and $Z_{b i} /\left|Z_{s r}\right|$ can be recognized as the real and imaginary part of the non-dimensional buoy impedance, respectively. It can be seen that a negative unconstrained optimal spring stiffness of the PTO system results from the imaginary part of the non-dimensional buoy impedance being smaller than 0.5 and the real part approaching 0.5 , and the extent of approaching is determined from the imaginary part. To avoid negative values, the spring stiffness of the PTO system should be constrained.

We selected a truncated circular cylinder as the reference underwater shape of the floating buoy as shown in Figure 2, where d denotes the draft and D for the diameter. The mass of the buoy is set as 
$m_{b}=\mu m_{s}$, in which $\mu$ is defined as the mass ratio. As for a simple case study here, we set the diameter to draft ratio $\gamma=D / d=2$, and $\mu=0.5$. Since the displacement of the buoy should be equal to the mass of both the offshore structure and the buoy, the dimension of the underwater part of the buoy can be obtained based on the mass of the offshore structure as

$$
\begin{gathered}
d=\sqrt[3]{\frac{4(1+\mu) m_{s}}{\pi \rho \gamma^{2}}} \\
D=d \gamma
\end{gathered}
$$

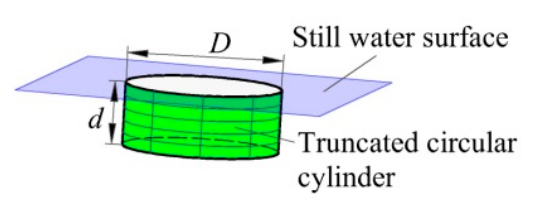

Figure 2. Diagram of the truncated circular cylinder.

Figure 3 shows the hydrodynamic coefficients and non-dimensional impedance of the buoy as a function of wave frequency. The hydrodynamic coefficients are calculated by the boundary element method (BEM) using the ANSYS AQWA software. Based on the hydrodynamic coefficients as shown in Figure 3a, the non-dimensional impedance of the buoy can be easily obtained as shown in Figure 3b. We find that, within the investigated range of wave frequency, the imaginary part of the non-dimensional buoy impedance is smaller than 0.5 , which implies that the unconstrained optimal spring stiffness of the PTO system is possible to be negative. Further, the real part of the non-dimensional buoy impedance decreases monotonously with wave frequency, and crosses 0.5 at $\omega=1.06 \mathrm{rad} / \mathrm{s}$. Therefore, within the wave frequency range determined from Equation (29), the unconstrained optimal spring stiffness of the PTO system will be negative.

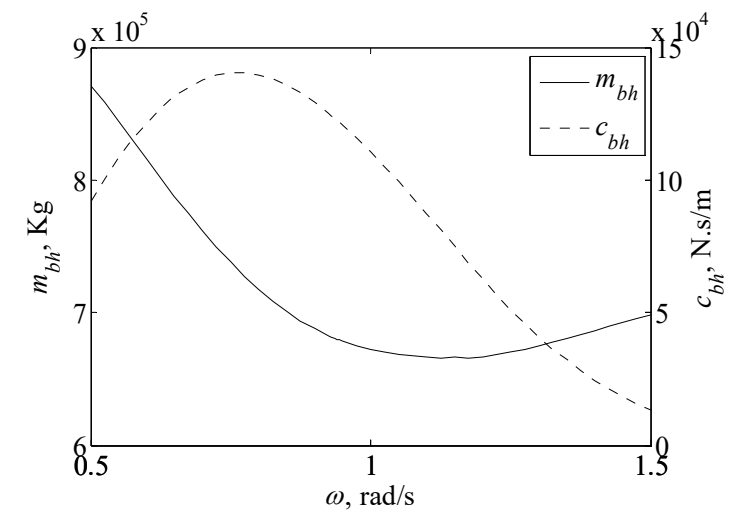

(a)

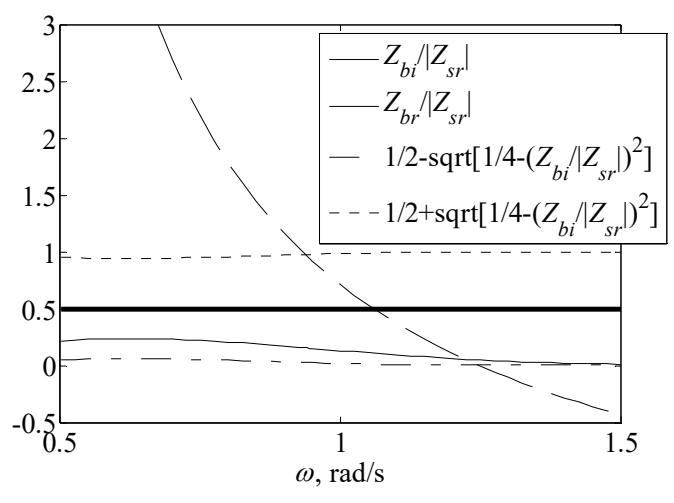

(b)

Figure 3. Hydrodynamic coefficients and non-dimensional impedance of the buoy as a function of wave frequency: (a) hydrodynamic coefficients; (b) non-dimensional impedance.

Figure 4 shows the optimal parameters of the PTO system and maximum absorbed power of the integrated system as a function of wave frequency. It can be clearly seen that, within the wave frequency range from $0.94 \mathrm{rad} / \mathrm{s}$ to $1.22 \mathrm{rad} / \mathrm{s}$, the constrained optimal spring stiffness of the PTO system is compulsorily tuned to 0 since the unconstrained optimal value is negative, as inferred from Figure 3 . Another obvious finding in Figure $4 \mathrm{a}$ is that, around $\omega=0.93 \mathrm{rad} / \mathrm{s}$, both the optimal spring stiffness and damping coefficient of the PTO system are impractically large, which can be attributed to the norm of $G$. The definition of $G$ can be found in Equation (10) and Equation (12). The imaginary part of $G$ is 
the negative of the imaginary part of the non-dimensional buoy impedance, while the real part of $G$ is the negative of the real part of the non-dimensional buoy impedance plus one. From Figure 3, we find that the imaginary part of the non-dimensional buoy impedance is much smaller in magnitude than the real part, indicating that the norm of $G$ is dominated by the real part. Around the wave frequency of $0.93 \mathrm{rad} / \mathrm{s}$, the real part of $G$ is crossing the zero point, which will result in impractically large values of $k_{p o}$ and $c_{p o}$ since the norm of $G$ is in the denominator of both. It is observed in Figure $4 \mathrm{~b}$ that the maximum absorbed power is less than the unconstrained case, which is denoted as $P_{\text {maxunconstrained }}$, within the frequency range when the constrained optimal spring stiffness of the PTO system is tuned to zero. This is as expected since the global extreme point of the absorbed power cannot be reached due to the constraint. This suggests that, to absorb the most wave power, the integrated two-body system should be carefully designed so that the wave frequency range where the constrained optimal spring stiffness of the PTO system is tuned to zero stays far away from the peak frequency of a spectrum, around which wave energy is concentrated.

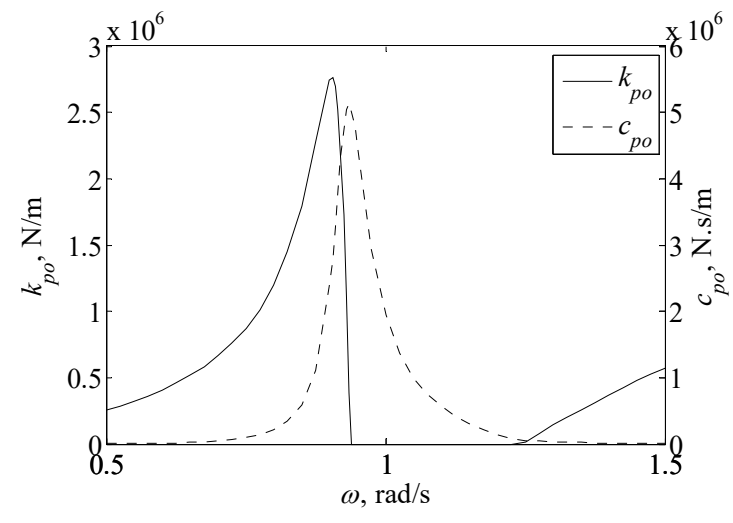

(a)

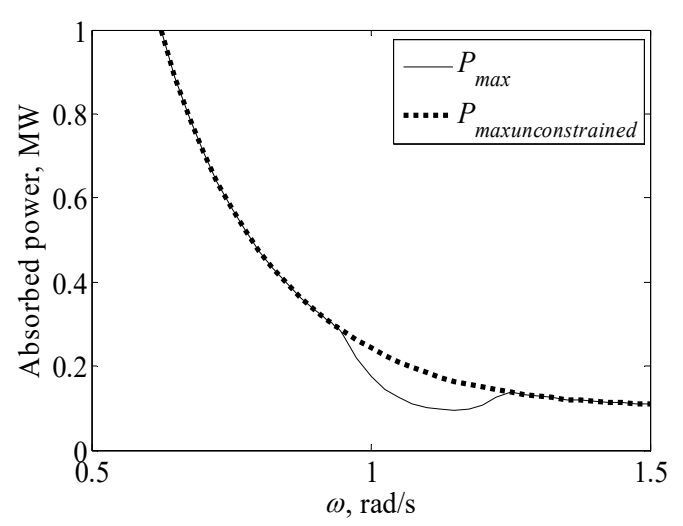

(b)

Figure 4. Optimal parameters of the PTO system and maximum absorbed power of the integrated system as a function of wave frequency: (a) Optimal parameters of the PTO system; (b) maximum absorbed power.

\section{Design of the Integrated System}

For a given offshore structure to achieve satisfied performance of the integrated system, parameters of the floating buoy should be properly designed. In this paper, parameters of the buoy designed include the mass and the underwater shape, while performance of the integrated system is focused on the absorbed wave power and motion amplitude of the two bodies.

\subsection{Design of the Mass of the Buoy}

In order to investigate the influence of the mass of the buoy on the performance of the integrated system, we fixed the underwater shape of the buoy while varying the mass. However, when varying the mass of the buoy, to support the varying total mass of the integrated system, the underwater shape will be changed accordingly to provide adequate displacement. Therefore, we take an alternative way to set the key feature of the underwater shape of the buoy to be fixed, and the diameter to draft ratio is chosen. Here, the underwater shape of the buoy employs the truncated circular cylinder, as shown in Figure 2, and the diameter to draft ratio is set to 2. It is assumed that the density of the buoy can be arbitrarily adjusted to fulfill the relation between the volume and mass of the buoy.

Figure 5 shows the optimal parameters of the PTO system as a function of wave frequency at different mass ratios. A general finding is that the maximum values of $k_{p o}$ and $c_{p o}$ decrease with the mass ratio, and the range of wave frequency when $k_{p o}$ is tuned to zero becomes narrower as well. Both 
suggest that the mass of the buoy should be larger to achieve better design of the PTO system, since smaller spring stiffness and damping coefficient seem easier to implement in engineering.

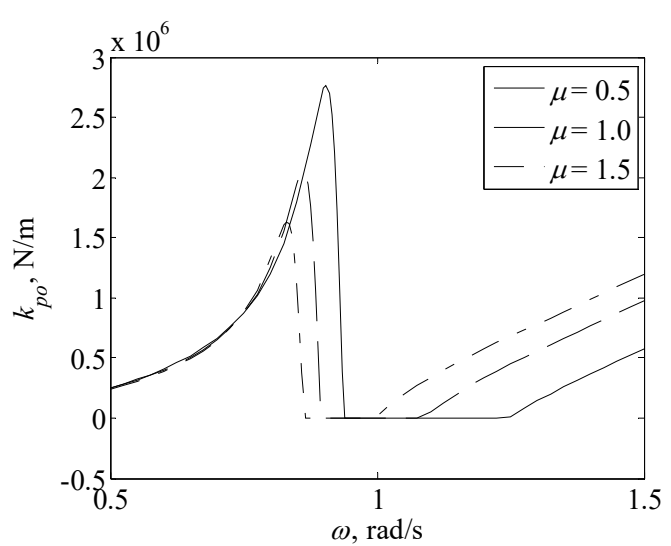

(a)

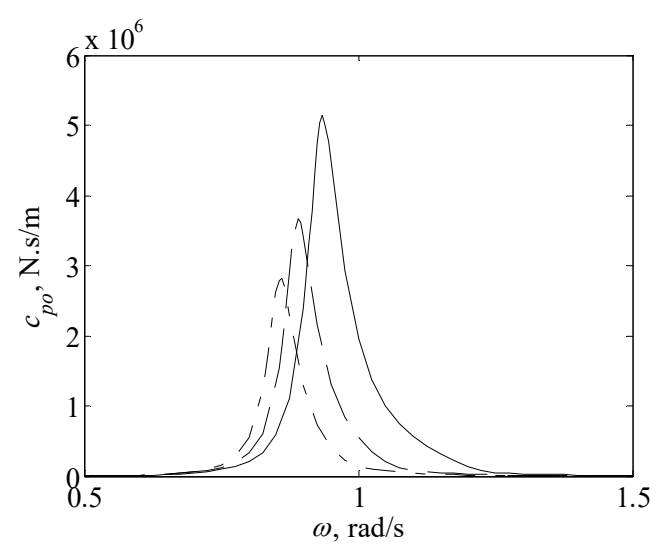

(b)

Figure 5. Optimal parameters of the PTO system as a function of wave frequency at different mass ratios: (a) Optimal spring stiffness; (b) Optimal damping coefficient.

Figure 6 shows the maximum absorbed power of the integrated system as a function of wave frequency at different mass ratios. A narrower range of wave frequency, where power absorption ability degrades due to the constraint on the spring stiffness of the PTO system, is observed at a larger mass ratio. Additionally, the absorbed power increases slightly with the mass ratio when the wave frequency is large. The above findings indicate that an integrated system with a larger mass ratio shows better power absorption performance.

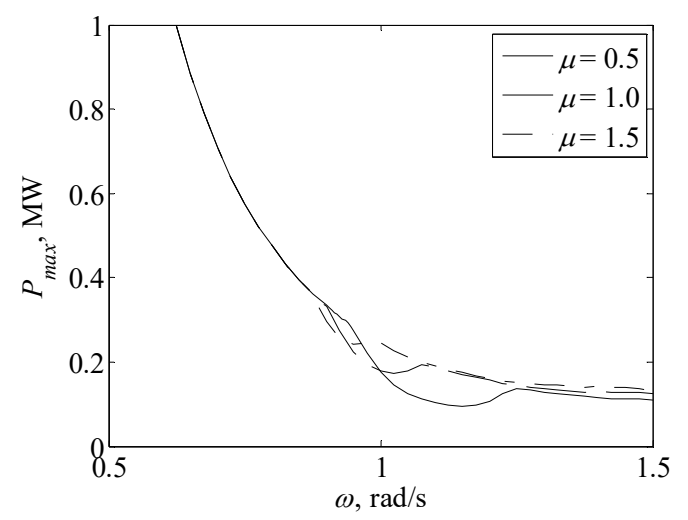

Figure 6. Maximum absorbed power of the integrated system as a function of wave frequency at different mass ratios.

Equation (7) gives the response of the offshore structure and the floating buoy respectively as

$$
\begin{aligned}
& \hat{z}_{s}=\frac{Z_{p} \hat{f}_{d}}{Z_{s} Z_{b}+\left(Z_{s}+Z_{b}\right) Z_{p}} \\
& \hat{z}_{b}=\frac{\left(Z_{s}+Z_{p}\right) \hat{f}_{d}}{Z_{s} Z_{b}+\left(Z_{s}+Z_{b}\right) Z_{p}} .
\end{aligned}
$$

Figure 7 shows the non-dimensional motion amplitude of the integrated system, which is defined by the motion amplitude divided by the wave amplitude imitating the definition of RAO as in [22], i.e., $z_{s n}=\left|\hat{z}_{s}\right| / A$ and $z_{b n}=\left|\hat{z}_{b}\right| / A$, as a function of wave frequency at different mass ratios. The 
non-dimensional motion amplitude of both the offshore structure and the floating buoy generally shows a tendency of fall first and then rise with the wave frequency. However, the motion amplitude of the buoy seems to be more sensitive to the constraint on the spring stiffness of the PTO system shown as the obvious reduction of the motion amplitude at corresponding wave frequencies, while the motion amplitude of the offshore structure seems to be only slightly influenced. A larger mass ratio generally shifts both curves of zsn and zbn to the left. Within the common range of wave period in the ocean, i.e., from $6 \mathrm{~s}$ to $10 \mathrm{~s}$ (wave frequency from $1.05 \mathrm{rad} / \mathrm{s}$ to $0.63 \mathrm{rad} / \mathrm{s}$ ), a larger mass ratio results in smaller motion amplitude of both the offshore structure and the floating buoy, which is preferred since smaller motion amplitude will not only reduce the influence on the function of the offshore structure due to the motion of itself, but also reduce viscous energy loss due to the motion of the buoy.

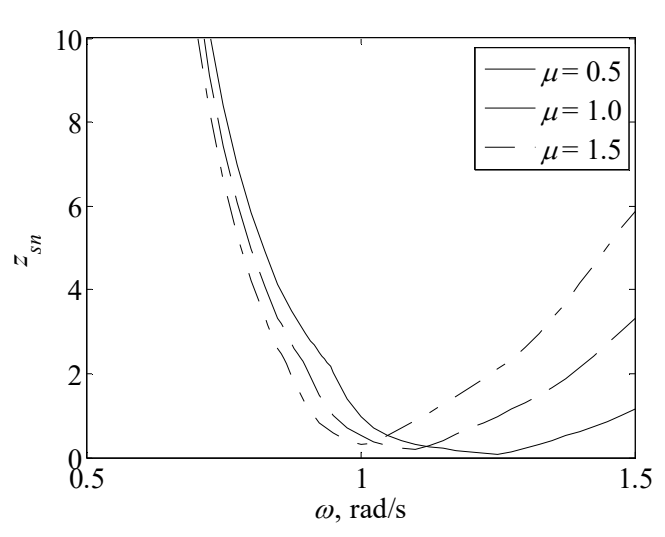

(a)

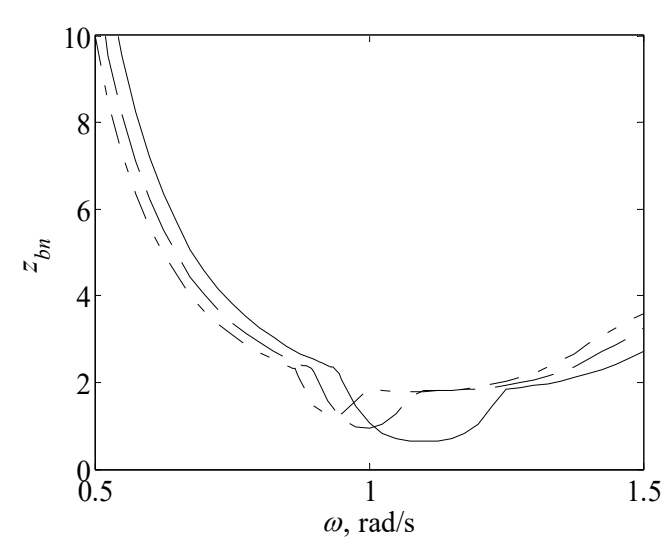

(b)

Figure 7. Non-dimensional motion amplitude of the integrated system as a function of wave frequency at different mass ratios: (a) For the offshore structure; (b) for the floating buoy.

Combined with the findings in above paragraphs, we conclude that a larger mass ratio is preferred for the integrated system due to smaller optimal parameters of the PTO system, more absorbed wave power, and less motion amplitude of the two bodies. However, in practical applications, the mass ratio should not be too large since it will introduce additional structural costs. Therefore, a balance between the performance of the integrated system and the structural cost should be made so that the overall profit reaches the maximum. In the following section, we set the mass ratio to be one for the designing process of the underwater shape of the buoy.

\subsection{Design of the Underwater Shape of the Buoy}

First of all, we focus on the influence of the diameter to draft ratio $\gamma$ of the underwater shape of the buoy. Figure 8 shows the maximum absorbed power and non-dimensional motion amplitude of the integrated system as a function of wave frequency at different diameter to draft ratios. At $\gamma=1$, the absorbed power is strongly influenced by the constraint on the spring stiffness of the PTO system, while at $\gamma=4$, the influence almost vanishes. Although smaller motion amplitude of the offshore structure from $0.76 \mathrm{rad} / \mathrm{s}$ to $1 \mathrm{rad} / \mathrm{s}$ is observed at $\gamma=1$, it is at the expense of the maximum absorbed power being significantly decreased due to the constraint. An overall comparison suggests that a larger diameter to draft ratio results in both more absorbed power and reasonable small motion amplitude of the two bodies. Therefore, we set the diameter to draft ratio to be 4 for further studies in the following. 


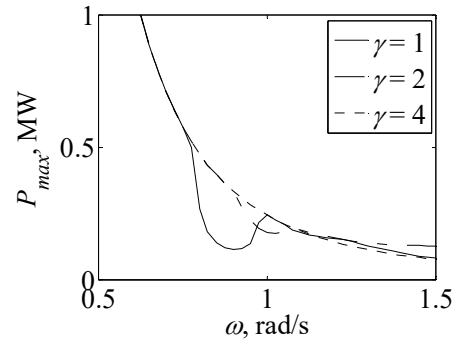

(a)

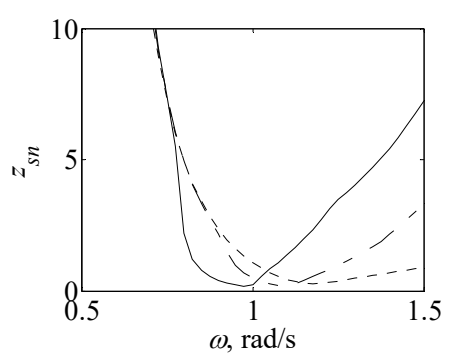

(b)

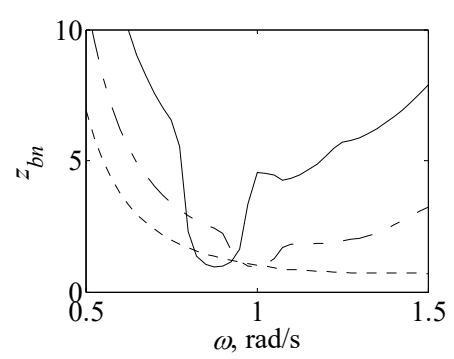

(c)

Figure 8. Maximum absorbed power and non-dimensional motion amplitude of the integrated system as a function of wave frequency at different diameter to draft ratios: (a) Maximum absorbed power; (b) non-dimensional motion amplitude of the offshore structure; (c) non-dimensional motion amplitude of the floating buoy.

Secondly, we focus on the influence of non-axisymmetry of the section of the underwater shape. Two representative non-axisymmetric section profiles, i.e., the equilateral triangle and the square as shown in Figure 9, are analyzed as opposed to the circle with axisymmetric section. In Figure 9, D represents the side length of the triangle and the square. Due to non-axisymmetry, the performance of the integrated system varies at different wave directions, which are defined by the wave angle $\beta$ in Figure 9. Here, we take two typical wave directions for both non-axisymmetric buoys into consideration, i.e., $0^{\circ}$ and $60^{\circ}$ for the triangle, and $0^{\circ}$ and $45^{\circ}$ for the square. Figure 10 shows the maximum absorbed power and non-dimensional motion amplitude of the integrated system as a function of wave frequency at different section profiles. An interesting finding is that non-axisymmetry of the section does not bring about significant influence on the performance of the integrated system, and only a slight increase of the motion amplitude of the buoy is observed for the triangular section. Here, the circular or square sections are recognized as the better choice for the integrated system.

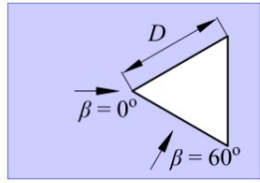

(a)

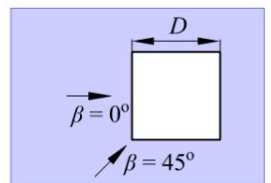

(b)

Figure 9. Two representative non-axisymmetric section profiles: (a) Equilateral triangular section; (b) square section.

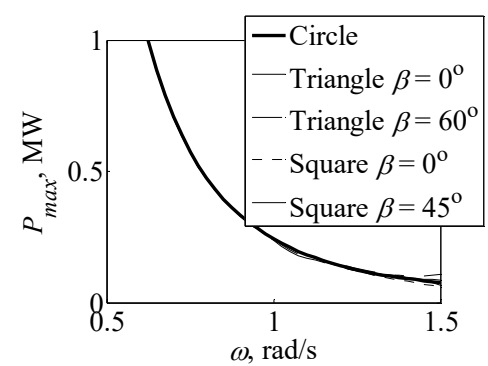

(a)

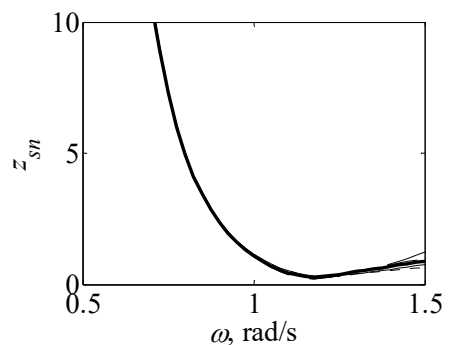

(b)

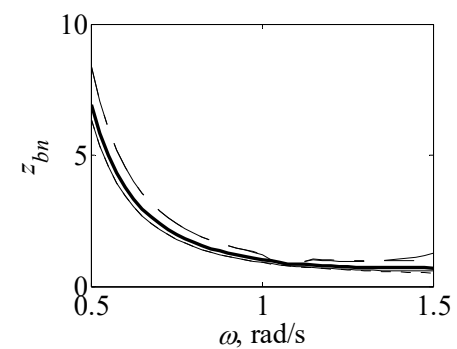

(c)

Figure 10. Maximum absorbed power and non-dimensional motion amplitude of the integrated system as a function of wave frequency at different section profiles: (a) Maximum absorbed power; (b) non-dimensional motion amplitude of the offshore structure; (c) non-dimensional motion amplitude of the floating buoy. 


\section{Performance of the Designed Integrated System in Irregular Waves}

The designing process in the previous section suggests that a larger mass of the buoy is better, and a favorable underwater shape of the buoy is characterized by large diameter to draft ratio and constant section size, with the section profile preferring a circle or square rather than an equilateral triangle. In this section, we progress the investigation into irregular waves to study the performance of the designed integrated system in real ocean sea. The mass ratio is set to 1 , and the truncated circular cylinder with a diameter to draft ratio of 4 is chosen as the underwater shape of the buoy.

An irregular wave can be recognized as the superposition of regular wave components [23] with the wave amplitude weighted by the energy distribution function, which in this paper employs the Pierson-Moskowitz spectrum [24], defined as

$$
S(\omega)=262.9 \frac{H_{s}^{2}}{\omega^{5} T_{e}^{4}} \exp \left(-\frac{1054}{\omega^{4} T_{e}^{4}}\right),
$$

where $H_{s}$ is the significant wave height and $T_{e}$ is the energy period. For the $n$-th regular wave component with frequency $\omega_{n}$, the wave amplitude is

$$
A_{n}=\sqrt{2 S\left(\omega_{n}\right) \Delta \omega}
$$

The response of the offshore structure and the floating buoy is given by

$$
\begin{aligned}
& z_{s}=\sum_{n} \operatorname{Re}\left\{\frac{Z_{p} \hat{f}_{d}\left(\omega_{n}\right)}{Z_{s}\left(\omega_{n}\right) Z_{b}\left(\omega_{n}\right)+\left[Z_{s}\left(\omega_{n}\right)+Z_{b}\left(\omega_{n}\right)\right] Z_{p}} \exp \left[\mathrm{i}\left(\omega_{n} t+\varphi_{n}\right)\right]\right\} ; \\
& z_{b}=\sum_{n} \operatorname{Re}\left\{\frac{\left[Z_{s}\left(\omega_{n}\right)+Z_{p}\right] \hat{f}_{d}\left(\omega_{n}\right)}{Z_{s}\left(\omega_{n}\right) Z_{b}\left(\omega_{n}\right)+\left[Z_{s}\left(\omega_{n}\right)+Z_{b}\left(\omega_{n}\right)\right] Z_{p}} \exp \left[\mathrm{i}\left(\omega_{n} t+\varphi_{n}\right)\right]\right\}
\end{aligned}
$$

where $t$ is the time, and $\varphi_{n}$ is the phase of the $n$-th regular wave designated randomly. The absorbed power of the integrated system is given as

$$
P=\frac{c_{p}}{t_{2}-t_{1}} \int_{t_{1}}^{t_{2}}\left(z_{s}-z_{b}\right)^{2} \mathrm{~d} t
$$

Due to orthogonality, influence of the coupled terms between different wave frequencies when inserting Equation (36) and Equation (37) into Equation (38) will vanish if the time interval between $t_{1}$ and $t_{2}$ is long enough [25]. Then, the absorbed power of the integrated system reduces to

$$
P=\sum_{n} \frac{\omega_{n}}{2} \frac{\left|\hat{f}_{d}\left(\omega_{n}\right)\right|^{2}}{\left|G\left(\omega_{n}\right)\right|^{2} Z_{p i}+2 Z_{b i}\left(\omega_{n}\right)+\frac{\left|G\left(\omega_{n}\right)\right|^{2} Z_{p r}^{2}+2\left[G_{r}\left(\omega_{n}\right) Z_{b r}\left(\omega_{n}\right)+G_{i}\left(\omega_{n}\right) Z_{b i}\left(\omega_{n}\right)\right] Z_{p r}+\left|Z_{b}\left(\omega_{n}\right)\right|^{2}}{Z_{p i}}}
$$

Figure 11 shows the contour of the absorbed power as a function of the spring stiffness and damping coefficient of the PTO system at $H_{s}=2 \mathrm{~m}$ and $T_{e}=8 \mathrm{~s}$. It is found that the absorbed power is a unimodal function of the spring stiffness and damping coefficient of the PTO system. As with previous sections, the spring stiffness of the PTO system should be constrained to be non-negative. Therefore, finding the maximum absorbed power of the integrated system is a constrained optimization problem, which is solved here by employing the fmincon function using the active-set method within the MATLAB environment. 


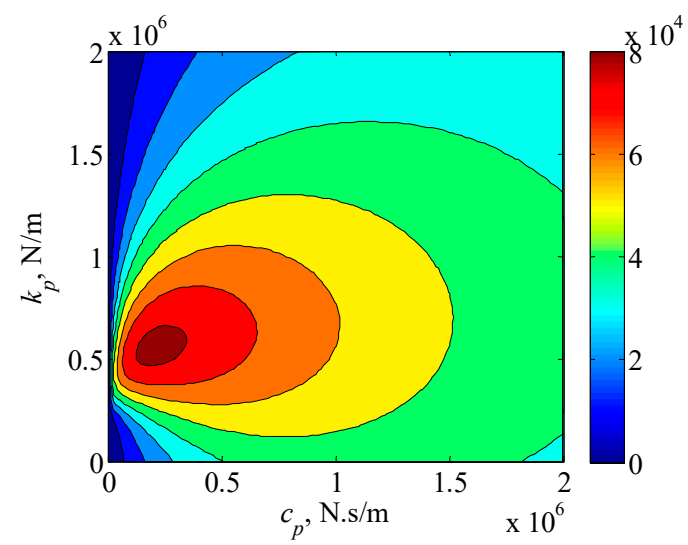

Figure 11. Contour of the absorbed power as a function of the spring stiffness and damping coefficient of the PTO system at $H_{s}=2 \mathrm{~m}$ and $T_{e}=8 \mathrm{~s}$.

Figure 12 shows the optimal parameters of the PTO system and maximum absorbed power of the integrated system as a function of energy period at $H_{s}=2 \mathrm{~m}$. It is found that the optimal spring stiffness of the PTO system automatically fulfills the constraint within the investigated range of energy period without being compulsorily tuned to zero. Both the optimal spring stiffness and damping coefficient experience rather milder variation with the energy period than in regular wave conditions, as shown in Figure 4; Figure 5. This can be explained by the fact that irregular waves are composed of wave components of different wave periods, and the behavior of the integrated system in irregular waves is the average of that in regular waves weighted by the energy distribution function. As a result, the violent variation tendency is filtered out by the averaging process. The maximum absorbed power peaks at the energy period of $7.3 \mathrm{~s}$, which is within the common range of wave period in the ocean, i.e., from $6 \mathrm{~s}$ to $10 \mathrm{~s}$, indicating that the power absorption ability of the integrated system is at the favorable state. When wave periods are larger than $10 \mathrm{~s}$, it is found that the curves in both subfigures of Figure 12 keep the current tendency and monotonically decrease with the wave period. Therefore, to focus on the WEC behavior in the common range of wave period, the data out of the range is not shown in the figure.

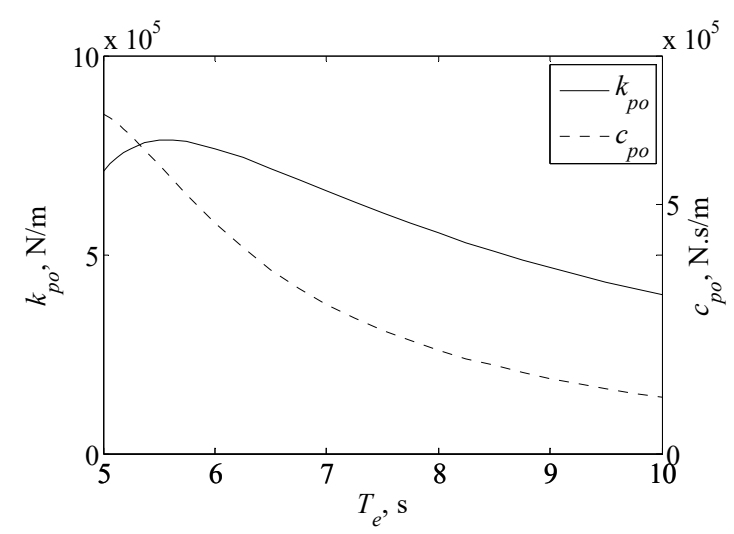

(a)

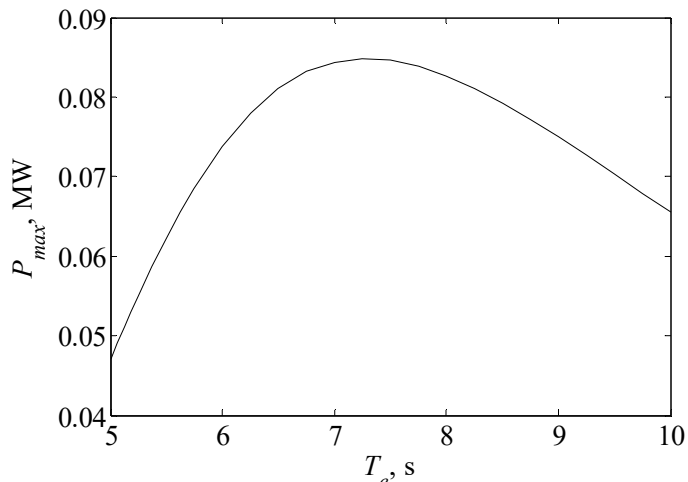

(b)

Figure 12. Optimal parameters of the PTO system and maximum absorbed power of the integrated system as a function of energy period at $H_{S}=2 \mathrm{~m}$ : (a) Optimal parameters of the PTO system; (b) maximum absorbed power of the integrated system.

Inserting the optimal parameters of the PTO system into Equation (36) and Equation (37) gives the motion response of the offshore structure and the floating buoy when the absorbed wave power is 
maximized. Figure 13 shows an example of the time history of the motion response of the integrated system at $T_{e}=6 \mathrm{~s}$ and $H_{s}=2 \mathrm{~m}$ when the absorbed wave power is maximized. It can be observed that the offshore structure always follows the motion of the buoy, but with a different magnitude and lag phase.

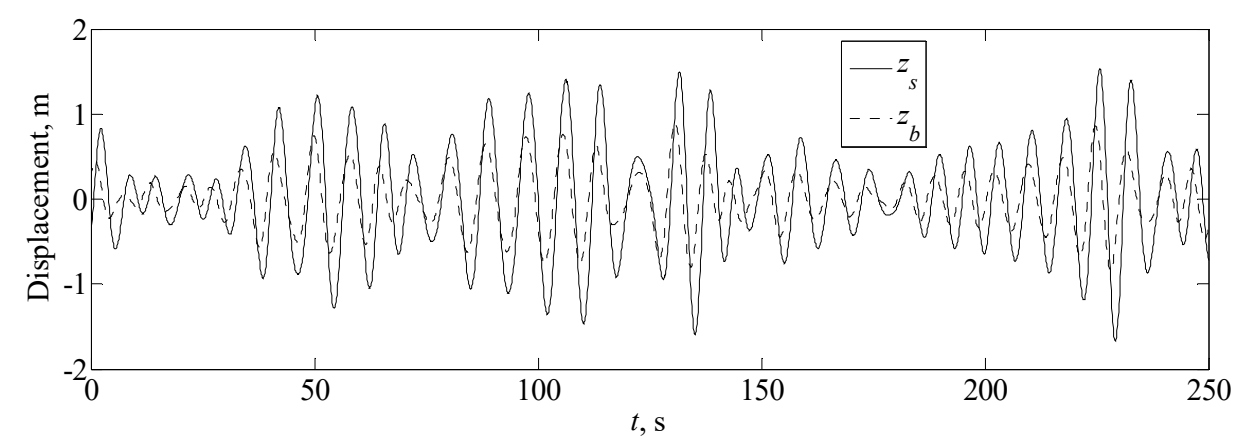

Figure 13. An example of the time history of the motion response of the integrated system at $T_{e}=6 \mathrm{~s}$ and $H_{s}=2 \mathrm{~m}$ when the absorbed wave power is maximized.

To quantitatively estimate the motion amplitude of the integrated system in irregular waves, we imitate the definition of the significant wave height, which is the mean of the highest one-third wave height in a wave record [26], to define the significant motion amplitude as the mean of largest one-third motion amplitude in a time history record. The significant motion amplitude of the offshore structure and the buoy are denoted by $z_{s s}$ and $z_{b s}$, respectively, and they probably represent the visual estimate of the motion amplitude of integrated system. As with previous representation of the motion amplitude of the integrated system, the non-dimensional form of $z_{s s}$ and $z_{b s}$ are respectively defined as

$$
\begin{aligned}
& z_{s s n}=\frac{z_{s s}}{H_{s} / 2} ; \\
& z_{b s n}=\frac{z_{b s}}{H_{s} / 2} .
\end{aligned}
$$

Figure 14 shows the non-dimensional significant motion amplitude of the integrated system as a function of energy period when the absorbed wave power is maximized. The calculated result is the mean of 10 different cases of random phases for the regular wave components. Within the investigated range of energy period, the motion amplitude of the offshore structure is larger than the buoy, and both increase with energy period. One may note that, in the designing process, we have tried our best to choose the design with smaller motion amplitude of the offshore structure, which however exceeds the wave amplitude for a certain range of energy period. This suggests that the offshore structure should be sophisticatedly designed to function insensitively to its heave motion. When offshore structures are not integrated with WECs, they are normally designed to suppress oscillation with waves. However, when they are integrated with WECs, their motion amplitude is even larger than waves. Therefore, to employ offshore structures to absorb wave power, functional devices in the offshore structure should work normally even when the structural support is under large oscillation. In this condition, measures may be taken to suppress oscillation of functional devices, such as motion compensation systems, vibration attenuation systems, etc. On the other hand, this is the accompanying price when the offshore structure is employed to absorb wave power.

Levelized costs of energy (LCOE) are defined as the ratio of total lifetime costs versus total expected outputs, and are used to measure economical competitiveness of a renewable energy technology. Total lifetime costs usually include investment costs (i.e., construction costs), operations, and maintenance costs. Since wave energy is still at an infant stage, lifetime costs of WECs vary significantly depending on many factors, such as the initial capital cost, the capture ratio, the discount rate, and the installation size. Some papers have done preliminary work on estimating levelized costs of wave energy. However, 
due to immaturity of WEC technology, the estimation process is usually based on many assumptions, and the estimated results show high level of uncertainty. For the integrated system in this paper, levelized costs of wave energy are also quite hard to estimate precisely. However, we can employ some basic data in the literature to provide a rough estimation to find the improvement of levelized costs of wave energy when WECs are integrated to offshore structures. It can be seen from [7] that levelized costs of wave energy are about $190 £ / \mathrm{MWh}$, in which the construction component occupies $155 £ / M W h$. When integrating WECs to offshore structures, the substructure, mooring system, and electrical grid connections can be shared. Therefore, when calculating levelized costs of wave energy of the integrated system, the above three parts that account for $36 \%$ of the construction cost [7] can be subtracted. As a result, the total construction costs reduce by 55.8 £/MWh. If we assume other costs beside construction to be consistent, levelized costs reduce by $29.4 \%$. Therefore, when integrating WECs with offshore structures, levelized costs of wave energy will be significantly reduced. However, we should note that the above calculation is quite simple and under a high level of uncertainty. Precise improvement of levelized costs of wave energy should take into consideration more factors and will be the work of next phase.

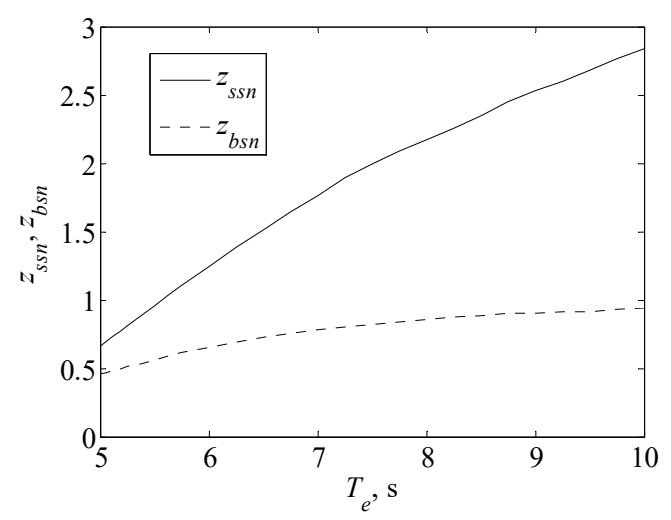

Figure 14. Non-dimensional significant motion amplitude of the integrated system as a function of energy period when the absorbed wave power is maximized.

\section{Conclusions}

In this work, our main interest is focused on the design of an integrated system, which consists of a floating buoy and an offshore structure on board and employs the relative motion between the two bodies to absorb wave energy, so that satisfied performance in the power absorption process can be achieved. The parameter to design is the mass and underwater shape of the buoy, and the performance concerned includes the absorbed power and motion amplitude of the two bodies. The spring stiffness of the power take-off (PTO) system is constrained to be non-negative with the concerns of complexity in implementation and system stability.

When PTO parameters are not constrained, the optimal spring stiffness will be negative when imaginary and real parts of the non-dimensional buoy impedance are smaller than and close to 0.5 , respectively. The constraint on the spring stiffness of the PTO system will reduce the maximum absorbed power when the unconstrained optimal value constrained is negative.

A larger mass of the buoy is advantageous due to smaller optimal spring stiffness and damping coefficient of the PTO system, more absorbed wave power, and less motion amplitude of the two bodies. The best underwater shape of the buoy is characterized by large diameter to draft ratio with the section profile prefers a circle or square rather than an equilateral triangle. Investigations on the designed buoy in irregular waves show that the integrated system presents its peak power absorption ability within the common range of energy period, and the motion amplitude of the offshore structure is larger than the wave amplitude in a certain range of sea states. 
Author Contributions: Conceptualization, Z.N.; formal analysis, Z.N.; methodology, J.W.; writing-original draft, J.W.; writing - review \& editing, J.W. All authors have read and agreed to the published version of the manuscript.

Funding: This research was funded by the NSFC of China, grant number 51905096, and the Basic Research Plan of Jiangsu Province, grant number BK20190373, and was also supported by “the Fundamental Research Funds for the Central Universities".

Conflicts of Interest: The authors declare no conflict of interest.

\section{References}

1. Buccino, M.; Stagonas, D.; Vicinanza, D.; Muller, G. Development of a composite sea wall wave energy converter system. Renew. Energy 2015, 81, 509-522. [CrossRef]

2. Foteinis, S.; Tsoutsos, T. Strategies to improve sustainability and offset the initial high capital expenditure of wave energy converters (WECs). Renew. Sustain. Energy Rev. 2017, 70, 775-785. [CrossRef]

3. Heath, T.V. A review of oscillating water columns. Philos. Trans. R. Soc. Lond. Ser. A 2012, 370, $235-245$. [CrossRef] [PubMed]

4. Oh, J.S.; Han, S.H. Inlet geometry effect of wave energy conversion system. J. Mech. Sci. Technol. 2012, 26, 2793-2798. [CrossRef]

5. Rebhi, R.; Ibala, A.; Masmoudi, A. On the modeling of a doubly-excited brushless claw pole alternator: Application to a solar-wind-wave hybrid energy conversion system. In Proceedings of the 8th International Conference and Exhibition on Ecological Vehicles and Renewable Energies (EVER), Monte Carlo, Monaco, 27-30 March 2013.

6. Astariz, S.; Iglesias, G. Output power smoothing and reduced downtime period by combined wind and wave energy farms. Energy 2016, 97, 69-81. [CrossRef]

7. Allan, G.; Gilmartin, M.; McGregor, P.; Swales, K. Levelised costs of Wave and Tidal energy in the UK: Cost competitiveness and the importance of "banded" Renewables Obligation Certificates. Energy Policy 2011, 39, 23-39. [CrossRef]

8. Liang, C.; Zuo, L. On the dynamics and design of a two-body wave energy converter. Renew. Energy 2017, 101, 265-274. [CrossRef]

9. Engström, J.; Kurupath, V.; Isberg, J.; Leijon, M. A resonant two body system for a point absorbing wave energy converter with direct-driven linear generator. J. Appl. Phys. 2011, 110, 124904. [CrossRef]

10. Korde, U.A. Systems of reactively loaded coupled oscillating bodies in wave energy conversion. Appl. Ocean Res. 2003, 25, 79-91. [CrossRef]

11. Parks, P.C. Wedges, plates, and waves: Some simple mathematical models for wave power machines. In Power from Sea Waves; Count, B.M., Ed.; Academic Press: London, UK, 1980.

12. French, M.J.; Bracewell, R.H. Heaving point absorbers reacting against an internal mass. In Hydrodynamics of Ocean-Wave Energy Utilization; Evans, D.V., Falcao, A.F., Eds.; Springer: Berlin, German, 1986.

13. Korde, U.A. On providing a reaction for efficient wave-energy absorption by floating bodies. Appl. Ocean Res. 1999, 21, 587-599. [CrossRef]

14. Zhang, H.; Xu, D.; Zhao, H.; Xia, S.; Wu, Y. Energy extraction of wave energy converters embedded in a very large modularized floating platform. Energy 2018, 158, 317-329. [CrossRef]

15. Chaplin, R.V.; French, M.J.; Bracewell, R.H. An improved version of PS Frog. In Proceedings of the 2nd European Wave Power Conference, Lisbon, Portugal, 8-10 November 1995.

16. Falnes, J. Wave-energy conversion through relative motion between two single-mode oscillating bodies. J. Offshore Mech. Arct. Eng. 1999, 121, 32-38. [CrossRef]

17. Ansys, Inc. AQWA Reference Manual; Version 16.0; Ansys, Inc.: Canonsburg, PA, USA, 2015.

18. Falnes, J. Ocean Waves and Oscillating Systems: Linear Interaction Including Wave-Energy Extraction; Cambridge University Press: Cambridge, UK, 2002.

19. Wu, J.; Yao, Y.; Zhou, L.; Chen, N.; Yu, H.; Li, W.; Göteman, M. Performance analysis of solo Duck wave energy converter arrays under motion constraints. Energy 2017, 139, 155-169. [CrossRef]

20. Cândido, J.J.; Justino, P.A.P.S. Modelling, control and Pontryagin Maximum Principle for a two-body wave energy device. Renew. Energy 2011, 36, 1545-1557. [CrossRef]

21. Roddier, D.; Cermelli, C.; Aubault, A.; Weinstein, A. WindFloat: A floating foundation for offshore wind turbines. J. Renew. Sustain. Energy 2010, 2, 033104. [CrossRef] 
22. López, M.; Taveira-Pinto, F.; Rosa-Santos, P. Influence of the power take-off characteristics on the performance of CECO wave energy converter. Energy 2017, 120, 686-697. [CrossRef]

23. Babarit, A.; Duclos, G.; Clément, A.H. Comparison of latching control strategies for a heaving wave energy device in random sea. Appl. Ocean Res. 2004, 26, 227-238. [CrossRef]

24. Vicente, P.C.; Falcão, A.F.; Gato, L.M.C.; Justino, P.A.P. Dynamics of arrays of floating point-absorber wave energy converters with inter-body and bottom slack-mooring connections. Appl. Ocean Res. 2009, 31, 267-281. [CrossRef]

25. Wu, J.; Yao, Y.; Zhou, L.; Göteman, M. Real-time latching control strategies for the solo Duck wave energy converter in irregular waves. Appl. Energy 2018, 222, 717-728. [CrossRef]

26. Holthuijsen, L.H. Waves in Oceanic and Coastal Waters; Cambridge University Press: Cambridge, UK, 2007.

(C) 2020 by the authors. Licensee MDPI, Basel, Switzerland. This article is an open access article distributed under the terms and conditions of the Creative Commons Attribution (CC BY) license (http://creativecommons.org/licenses/by/4.0/). 